\title{
Towards a Social Internet of Things Enabled Framework for Supply Community Networks
}

\author{
Mohamed Omar ${ }^{1}$, Paul Mason ${ }^{2}$ \\ ${ }^{1}$ PhD Student, School of Science \& Technology, Shinawatra University, Pathum Thani, Thailand \\ ${ }^{2}$ School of Science \& Technology, Shinawatra University, Pathum Thani, Thailand \\ Correspondence: Paul Mason, School of Science \& Technology, Shinawatra University, Pathum Thani, Thailand.
}

Received: November 18, 2019

Accepted: December 5, 2019 Online Published: December 12, 2019

doi:10.5539/ibr.v13n1p121

URL: https://doi.org/10.5539/ibr.v13n1p121

\begin{abstract}
Social Internet of Things (SIoT) is one of several emerging internet paradigms, signaling the inevitable fusion of Internet of Things with Social Networks. This paper demonstrates the feasibility of applying an existing SIoT framework to Supply Community Networks (SCN). a term we use to describe the changing pattern of supply chains whose morphology continues to evolve from traditional linear continuums, into ostensibly mesh-like structures. Specifically, we identify an appropriate SIoT architecture from current literature which was used as a basis for realizing the notion of SCN, where in this case the 'members' are autonomous objects (Supply Community Agents, or SCA) working on behalf of member organizations (suppliers, manufacturers, retailers, etc.) and whose generic object architecture we extended by specifying interfaces to various member functions that all such agents must possess to engage in the exchange of goods/services information and remittance one would expect whether part of a chain or as here, a network (or networks). We substantiate our claims of feasibility using stochastic MATLAB simulation of a baked-goods SCN scenario. Results showed that the modified SIoT framework exhibited the flexibility required by SCAs when operating as part of a Supply Community Network so that they can effectively discharge their responsibilities in delivering the services needed by other member agents of a SCN.
\end{abstract}

Keywords: Supply Community Networks, Social Internet of Things, Social Networks

\section{Introduction}

To say that the corporations today conduct businesses in a massively changing environment is to grossly understate the issue. Brian Solis (Solis, 2015), formerly a principal analyst with Altimeter Group coined the phrase "digital Darwinism" to capture the essence of this phenomenon, one where technology and society are evolving faster than businesses can naturally adapt; where computers have contracted transaction times into units so small they exceed the realms of human consciousness; where brainpower is amplified in ways comparable to that in which steam engines, metallurgy and giant power tools swelled muscle power during the Industrial Revolution (Rizk, 2002); where trading is now routinely performed by algorithms because humans are simply too slow (a microchip can identify, prepare and close a trade in 740 billionths of a second!) Thus, with our economies pumped on steroids, rival companies are caught in a 'pitched battle' with competitors to lower latencies of each component in their already sophisticated set-ups and thereby gain competitive advantage. Lapsed time between completion of one exchange and introduction of the next represents the kinds of lost productivity and added cost of doing business in traditional supply chains that eventually makes markets obsolete.

Supply Chains and their Management (SCM) are now being powered by new communication technologies backed by massive cooperative efforts that facilitate absolute process integration; this was underscored by a previous study (Omar \& Mason, 2019) in which we proposed a taxonomy of all possible flow dimensions, preconditions necessary to render such a scenario possible. We also noted a seismic shift in the organization of supply chains whose morphology continues to evolve from rigid linear continuums, into what are ostensibly omni-channel, mesh-like structures dubbed Supply Community Networks (SCN). A SCN, to borrow from James F. Moore, is a business ecosystem (Moore, 1996); an interconnected grouping of biotic elements and abiotic components (respectively all stakeholders and all non-living phenomena including but not limited to organizations, plant, (information) technology, commodities, and remittance) engaged in the production and 
delivery of goods and services of value to customers, customers who are themselves SCN members (Omar \& Mason, 2019).

A strong cross-pollination of ideas exists between Supply Community Networks and Social Network theory (Freeman, Roeder, \& Mulholland, 1979); particularly striking are similarities to Granovetter's seminal piece on the notion of strong and weak ties (Granovetter, 1973). This paper ventures further however, by seeking to explore the possibility of replacing humanoid SCN 'members' with smart, autonomous (trust-based) objects (herein dubbed Supply Community Agents, or SCA) working on behalf (or rather taking the place) of, operatives from said member organizations (from suppliers to producers, consumers to decomposers, etc.).

To make our hypothesis fly, and hence demonstrate proof of concept, we turned to the Social Internet of Things (SIoT). SIoT is one of several emerging internet paradigms ${ }^{1}$, signaling the inevitable fusion of Internet of Things (IoT) with Social Networks to form socio-technical networks. While the Internet is limited to the exchange of data and documents of various media types, IoT addresses networking of everyday objects; from large objects (cars); to small objects (wearables); and often bizarre objects (connected rectal thermometers anyone?). In other words, IoT instituted an amalgamation of the digital and physical worlds. Decisively however relationships among connected objects, while not necessarily persistent, are pre-configured such that cardinality of nodes is fixed (even if non-autonomously extensible). Conversely SIoT has anthropomorphized (i.e. socially-oriented) objects by engineering-in features fostering autonomous ${ }^{2}$ 'friend' discovery (and thus social-group formation), as well as selection and composition of services and information potentially provided by any distributed objects and networks that have access to the physical world (Paulo, 2011), (Atzori, Iera, \& Morabito, Siot: Giving a social structure to the internet of things, 2011), (Nitti, Roberto, Atzori, Iera, \& Morabito, 2012) and (Atzori, Iera, Morabito, \& Nitti, The social internet of things (siot)-when social networks meet the internet of things: Concept, architecture and network characterization, 2012). Note a friend in our present context implies any trading partner who cooperates towards mutually beneficial goals in certain domains, but who simultaneously serves their own interests by actively competing in others (sometimes referred to as coopetition (Luo, 2007) and (Ghobadi \& D'Ambra, 2012)).

The last point is immensely significant and worthy of further attention. Levels of coopetition are shaped by the prevailing business environment facing SCA, from navigating calmer waters to battling the perfect storm. Moore [ibid] used several ecological metaphors, suggesting that any firm is embedded in a (business) environment; that it must co-evolve with other companies in that environment, and that "the particular niche a business occupies is challenged by newly arriving 'species' (with their 'substitute products/services' (Porter, 1979)). Thus, sometimes a community will be unified a la Friske's theory of social relations (Fiske, 1992) communal sharing, equality matching, authority ranking and market pricing - which is congruent with the vision for SIoT set out in (Atzori et. Al., 2012). However, we contend that when market brutality dictates, organizations, or rather strategists running them, become asocial (Blau, 1964 ); where social relationships become an instrumental means towards extrinsic, non-social ends, i.e. constraints towards satisfaction of an SCN sub-collective's 'individual' desires (readers a referred to (Luo, 2007) for an in depth discussion factors influencing coopetition).

When SIoT first emerged, it represented a field of pure research. Some might argue it still is; since entering the Internet lexicon, the language of publications remains a "future vision" with little by way of empirical evidence. In section two we revisit one such corpus and give the architecture described a credible outlet by applying it to our notion of Supply Community Networks. Note, however that while theoretical works on Social Internet of Things have hitherto emphasized only positives - a means of cultivating relationships on behalf of human mediators, but without their intervention and to the ultimate betterment and wellbeing of individuals, rganizations, sectors and society - we realize that objects in the commercial world must be endowed with both social and asocial behavior which itself is shaped by the values of those who prime (program) them. This we stress has no impact on the architecture itself, only the way it is used.

\footnotetext{
${ }^{1}$ While the number of Internet connected devices is expected to exceed 50 billion by 2020, the IoT revolution is still in its early throes (Gordon, 2018). Equally arresting is that IoT is the outcome of more than half a century of internet evolution (taking Licklider's (Licklider, 2001) "Galactic Network" concept as our baseline); so, in the here-and-now it may seem to be its culmination. Not so. In the words of Astronomer Royal Martin Rees, the internet 50 years hence will be in every which way different from today, as humans are from bacteria. In time we believe IoT will either diverge along many branches, each one tied to a specific environmental deployment, or as seems more plausible, that all affixes will eventually disappear leaving us once again with a 'vanilla' internet.

${ }^{2}$ Meaning without human intervention
} 
The remaining sections of this paper are structured as follows. Section 2 illustrates how the notion of Supply Community Agents and Supply Community Networks can be realized based on principles described in current SIoT literature, before we place this work in context using a hypothetical narrative (scenario) to be used for evaluation; Section 3 describes that evaluation using MATLAB simulation along with our key findings and results; finally Section 4 sets forth some conclusions and future research agenda.

\section{Foundations \& Fundamentals; Context \& Characterization}

In this section we introduce the architectural foundations on which Supply Community Agents (and hence SCN) are based. A contextual narrative (and exemplar characterization) to be used for evaluation in Section 3 are also introduced to concretize the principles described.

\subsection{Foundations \& Fundamentals: Towards Realization of Supply Community Agents}

Having judiciously hypothesized a common denominator exists between our notion of Supply Community Networks and their potential realization using SIoT, we undertook a 'systematic' literature survey (SLR) (Kitchenham, et al., 2009) of the latter. In academia, SLR are the recommended method for aggregating evidence, in this case to support a tentative argument - on a (still) subterranean subject-matter - before committing to further work. Our aim, to state answers to three questions: i) who leads the field in this sphere of research; ii) what research topics are being addressed of SIoT; and iii) what limitations exist in the afore-said research?

Our findings were as follows: unquestionably the pioneer, doyen and torchbearer for SIoT has been Luigi Atzori from the Department of Electrical and Electronic Engineering at University of Cagliari; Atzori has been a prolific contributor to the field, with no less than thirty-three publications dating back to 2011, racking up in excess of 2,750 citations (based on Google Scholar statistics). A variety of research topics feature in the literature, including concepts, architectures, platforms, network infrastructures, trustworthiness, friend selection, navigability, cloud-based SIoT and mobile-crowdsensing have all are come under the microscope. However, the striking thing is that with a few exceptions (see for example (Girau, Nitti, \& Atzori, 2013)) the overriding theme is of one of potential, potential, potential. Potential applications; potential benefits to individuals, organizations, sectors and societies; and potential dangers in smart objects mimicking human-like personality in designing smart-objects (Pieroni, et al., 2015).

The literature, in particular works authored (or co-authored) by Atzori provided a promising foundation for further for realizing our notion of Supply Community Networks. As such SCA establish social relationships with one another autonomously based on appropriate policies (rules). Below we describe the functional components as a possible architectural model for SIoT objects followed by the systems architecture.

\subsubsection{Objects Functionalities}

Atzori et. al., 2012 identified appropriate features and functionalities for the establishment and management of social relationships between objects (again in our proposed context this implies SCA) in such a way that they are navigable when integrated into a social network context (Figure 1). Central to every object are the functions of ID management, object profiling, and owner control. Assigning an ID to an object universally identifies all possible categories of objects. Object profiling meanwhile functions to determine static and dynamic information about objects, while owner control enables the identification of activities that can be performed by an object; e.g., information that can be shared with other objects or the type(s) of relationship to be established (with another object).

In addition, they identified further components deemed necessary in an object, including service discovery that enables objects to seek out other objects that can provide whatever services are required, when they are required (again the parallels with SCM chime with our view of fundamentally synergistic agents favoring strong ties, but with the capacity to become self-serving whenever needs dictate) (Omar \& Mason, 2019). Next up, the relationship management function allows an object to start, update and terminate relations with other objects, whereas service composition enables interactions between objects. This relates either to retrieving information about the 'real world', or finding services from other objects.

Trustworthiness is vital both in business and in social networking. The trustworthiness management component is therefore able to understand how information provided by other objects needs to be processed; reliability is based on the behavior of other objects and is strictly related to the relationship management feature. (Nitti, Girau, \& Atzori, Trustworthiness management in the social internet of things, 2013) defined two models of trustworthiness; the first, based on own experience, where every node computes the dependability of its 'friends' and opinion of friends in common with potential service providers. Secondly, using a structure of distributed hash tables, information regarding each node is stored and distributed with the intention that any node can use similar 
information. Finally, the APIs service component is comparable to that required in social networking systems as an interface to any SIoT network.

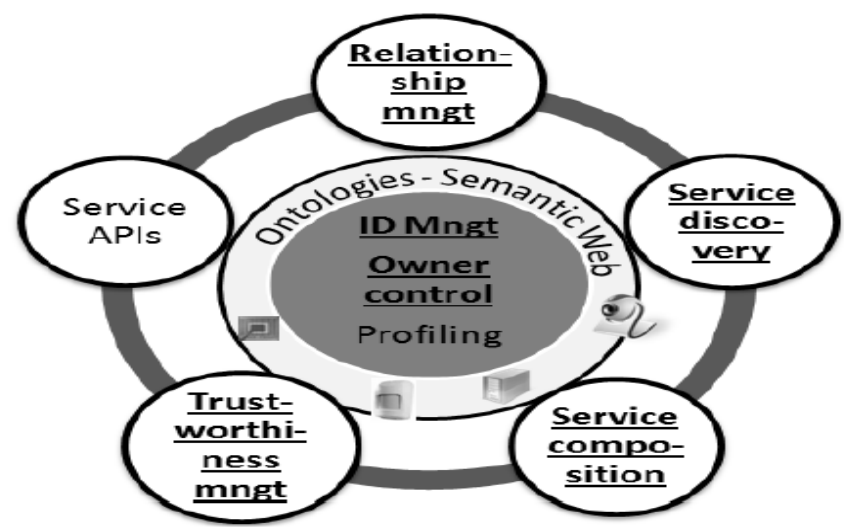

Figure 1. Features and Functionalities of Smart object (s) (source: Atzori et. al., 2012)

\subsubsection{System Architecture}

Atzori et. al., 2012 also proposed a system architecture for realizing the SIoT concept comprising: a sensing layer devoted to data acquisition and node collaborations in short range and local networks (i.e. between SCA within a SCA); a network layer aimed at transferring data across different networks (i.e. between SCAs); and an application layer used to deploy IoT apps together with middleware functionalities.

The architecture is described both from the server side and object side which may differ in terms of encompassing layers. The server side includes the network and application layers; the application layer itself contains sub layers including a database and metadata on member profiles, activities carried out by an object and descriptions about the data itself. Another database stores relevant ontologies of social activities as a semantic view; semantic search engines are used to extract this view.

The object side architecture may vary depending on the characteristics of an object. If an object is a dummy with the lowest functionality (e.g. it either has an RFID tag or sensing device) whose sole purpose is sending signals to other object' gateway, then said gateway needs to be equipped with the whole set of layer functionalities. However, if an object can detect the physical world data and transmit over an IP network, then network functionality will be assigned to that object; the gateway application layer functionality would be sufficient. In addition, if an object is smart (e.g. a smart phone) it may implement the functionality of all three layers such that no gateway is required. The layered architecture is shown in Figure 2.

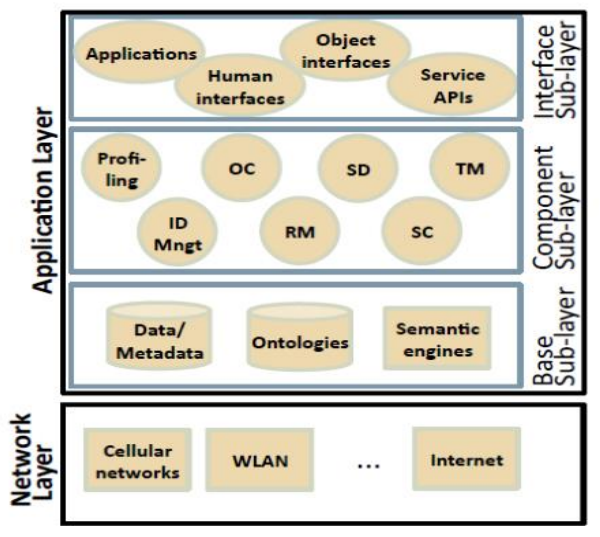

SIOT SERVER
GATEWAY

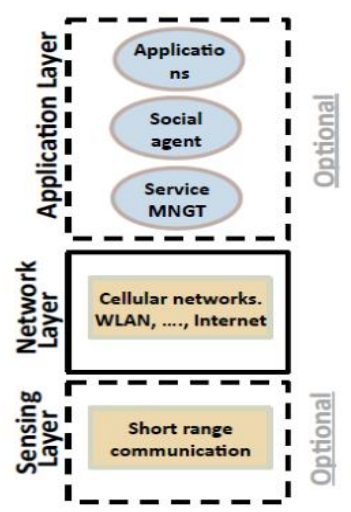

OBJECT

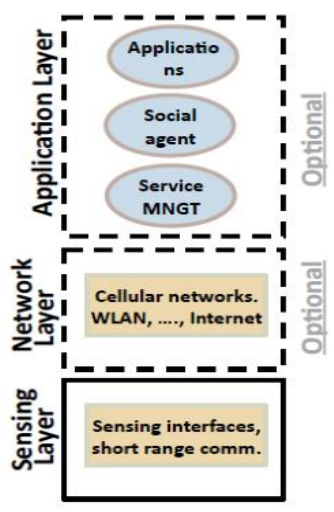

Figure 2. Layers of the Systems Architecture (source: Atzori et. al., 2012) 
While the work outlined above is sufficient inasmuch as a number of application areas could potentially utilize the solution providing new services to users, the authors did not explore it in a particular context. While (Turcu \& Turcu, 2017), presented a future vision for healthcare, the generic functions of SIoT need to be fleshed out according to the context in which they are to be applied.

We express these functions (methods) below as they show how SCA interact across a SCN. Note the volume of data generated by SCA are each stored at the base sub-layer in application layer and be achieved without human intervention. These functionalities are described and defined below in terms of their input parameters and return values:

Table 1. SCA functions (methods)

Boolean Can_Service Service_Discovery (Integer Service_Discovery takes input parameters Quantity and Quantity, Money MaxPrice)

MaxPrice returning a Boolean value of Can_Service.

For instance, an agent accesses a Supply Community Network to locate possible agents able to accommodate their preferences such as the number of required products, the maximum affordable price, and required date and time of the needed products.

Boolean Is_Trustworthy TrustMgt (Integer ID, Text TrustMgt reads information regarding each Node/Agent Name, Boolean Have_Relations) e.g., ID, Name, Relationships

from a distributed hash table to understand and establish trust with other Agents and returns whether the Node/agent is trustworthy or not.

\begin{tabular}{lll}
\hline Enum Decision RelationshipMgt & (Integer ID, Text & RelationshipMgt reads information regarding each \\
Name, Boolean Is_Trustworthy) & Node/Agent, returning an enumerated type - a \\
& $\begin{array}{l}\text { decision that allows a SCA to start, update or terminate a } \\
\text { relation. }\end{array}$
\end{tabular}

For instance, a manufacturer agent reads a supplier's information such as ID, Name, Is_Trustworthy as True then reaches a 'relationship decision with that agent.

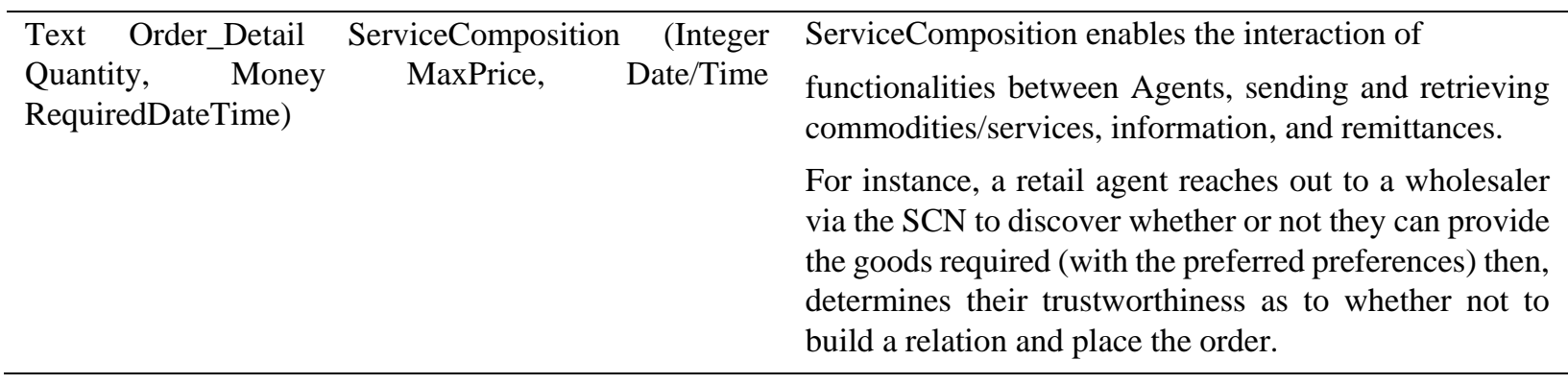

\subsection{Context \& Characterization}

In our study we demonstrate the working practice of SCN using stochastic simulation (Huynh \& Soumare, 2011). Simulation can be used in a range of applications, from operations research to project management, reliability engineering to economics. Simulation is also a valuable tool to test proposed strategies in unpredictable environments such as multi-tier networks. It can represent discrete events and incorporates uncertainties. For instance, when the store of a retailer reaches its minimum stock level, a purchase order is triggered; if the replenishment did not occur as planned, it jeopardizes relations with other agents selling out of stock products. So, to demonstrate and analyze SCN we first compiled a narrative (Scenario); we then use MATLAB/Simulink (Kattan, 
2008) as our means of implementation, thereby demonstrating how the services provided and consumed by SCA both individually and collectively are enacted.

\subsubsection{Supply Community Network Context}

Consider a hypothetical SCN for baked goods that starts with suppliers of raw ingredients (materials) and ends with delivery of the product to the end consumer. As with any industry, baked goods SCN are subject to numerous variations; so, to circumvent all possible end to end perambulations, we borrow from (Mack, 2018). Where possible however, conceivable variations are highlighted.

Raw ingredients are often supplied directly to baked goods manufacturers from farmers or possibly agricultural cooperatives. Flour, sugar, wheat and other commodities are generally interchangeable (notwithstanding quality variations), so a manufacturer may buy from whichever supplier offers the lowest prices. However, other ingredients, such as flavorings or special types of flour, might not be widely available, meaning a manufacturer must seek out affordable suppliers.

At the manufacturing stage of a SCN, a baked goods company converts the raw ingredients into a product, such as bread, cookies, cakes, patisseries and many other items. As previously indicated, the ensuing manufacturing processes will depend entirely on each given company's plans for the downstream stages. If for example the baked goods are to be shipped worldwide, preservatives must be added during the manufacturing process. Alternatively, if the goods will be packaged for home preparation, an entirely different production process will unfold.

The distribution stage involves moving products from the manufacturing production facility to wherever the consumers are. Distribution can be simple, or it can involve many distinct channels. For example, a baked goods company might sell its wares directly to consumers through a factory outlet and its website, as well as to wholesalers. Wholesalers are merchants that buy products from manufacturers and use their expertise in shipping and distribution to re-sell the products to retail stores.

Delivery of the products to consumers marks the final stage in this (or any other) SCN. Most consumers buy baked goods from a retail store, such as a supermarket or specialist bakery (again purchases may be online with home delivery). The retail store can promote such products in a variety of ways, such as choosing a visible position on its shelves or by offering samples to customers. Such promotions may or may not impact significantly on demand and therefore may or may not affect SCN activity.

\subsubsection{Characterization Walkthrough}

Modeling the above narrative, we assumed a number of agents including suppliers, manufacturers, distributors, wholesalers, retailers and consumers. Each SCA is characterized by both the generic functionalities set out in Atzori et. al., 2012, together with specific SCN features described in 2.1.2. SCA also possess and are built using the above SIoT architecture in terms of their servers and other objects to realize social networking behavior. These SCAs establish social relationships with other SCA autonomously based on policies (rules) described in Section 2.

Thus, we now demonstrate how these agents locate one another so as to accommodate their preferences (e.g. place orders) using SIoT features. Also, we describe how an agent (e.g., a retailer) can employ dynamic pricing to vary the cost of a particular product based on demand. Furthermore, we discuss how agents may locate each other to join forces when placing orders to reduce costs, before finding and appropriate agent to accommodate their requirements. Therefore, this demonstration reflects the bounds of flow possible in the SCN identified in (Omar \& Mason, 2019) applying the order fulfillment process shown in Figure 3. Note, here the focus is on a single SCN and its intra-relationships as communication of SCA between supply community networks (inter-relationships) while on the rise - remains less common and would merely replicate the same principles.

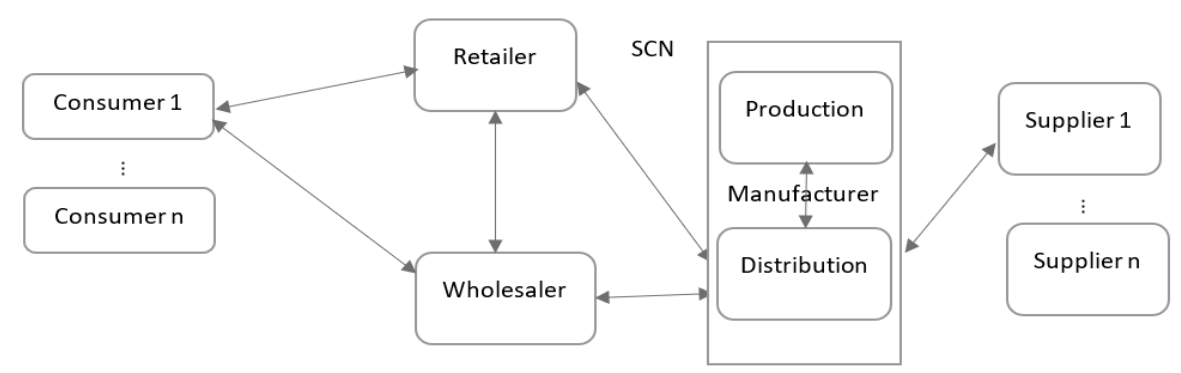

Figure 3. SCA Characterized in SCN 
Using a consumer object (app) on their smart phone, wearable or other smart device consumer agents access the SCN to locate possible retailers able to accommodate their preferences and thereupon place an order. Features like service discovery, relationship management, trustworthiness, and service composition within the sensing, network and application layers enable him or her to identify their preferred agent (retail store) from which to purchase baked goods. Consumers may trade off store proximity against price of goods in reaching a decision; Using the service composition functionality, the store's retailing system interacts with smart shelves, featuring embedded sensors to locate the ordered items (thereby freeing up manpower to focus on other duties). The store shelves then interact with store staff - equipped with pick-by-vision glasses - by sending notifications about consumer orders which are then prepared for shipment. When assembled for dispatch, staffs communicate with the logistics agent (object) to inform them that a delivery is queued. By extracting real time information using the semantic view, the logistics agent-object detects whether that delivery can be made and if not requests reschedule.

In this case, the retailer buys baked goods either directly from a manufacturer's distribution center or else from a wholesaler (who also buys their products from the manufacturer's distribution center). Interacting with the store's smart shelves, the retail store analyses the semantic view to examine item stock levels. If necessary, the retail store agent object reaches out via the SCN to discover a manufacturer distribution center or wholesaler that can provide the baked goods required (with the preferred preferences). Once located, the order is placed using SIoT functionalities like service discovery, relationship management, trustworthiness, and service composition via the sensing, network and application layers. Moreover, given that baked goods are perishable, so the retailer's shelves smartly communicate to staff signaling any inedible and/or expired products to be either thrown away or else relocated when approaching their expiry date.

When a wholesaler buys produce from the manufacturer's distribution center, it does so in response to on hand stock insufficiencies identified by analyzing the sensor and engine data and also from customer needs. Moving products from the manufacturing production facility to wherever the consumers are, the machinery systems first prepare and package the baked goods; then, the distribution agent analyses the SC semantic view to retrieve information (new production and/or consumer orders) requiring dispatch. This involves using the service composition function for object interaction and the sensing layer with devoted data acquisition and node collaborations in local (internal) networks in addition to semantic engines that are used to extract information.

The manufacturing agent converts raw ingredients into baked goods, scheduling production around orders received and current stock on hand. If stock is insufficient, caused for example by a sudden unexpected order spike, a schedule agent updates the manufacturer agent which in turn may reprogram production machinery (e.g. dough mixers, cutters, oven-feed conveyors and of course ovens). Also, for smooth operation at the production side for instance, the sensing layer and semantic engine allows sensing and detection of real-time conditions, analyzing such data to anticipate machinery failures and other delay problems before they happen; or to reschedule production and update other agents.

When the manufacturer's warehouse is in need of replenishment, it seeks out and then sends a purchase order for the raw ingredients required to a supplier - in this case farms or agricultural cooperatives who can satisfy their preferences, or to be more precise, SIoT functionalities (service discovery, relationship management, trustworthiness, and service composition, together with the sensing, network and application layers) are used to canvas the SCN to leverage the best deal since, as stated previously, the manufacturer can be selective with suppliers because many raw ingredients are interchangeable. The chosen supplier(s) then provide the required ingredients and undertakes distribution. Responding to received orders from a manufacturer, the distribution agent analyses the required order to arrange dispatch, communicating with the transportation system to complete delivery.

\section{Results and Discussions}

Using MATLAB, we now demonstrate how SCA seek out other agents able to accommodate their order preferences using the SIoT object functions and architecture described in Section 2. We further show how agents can employ dynamic pricing ( $\mathrm{Li}$, Zhang, \& Han, 2017) strategies in accordance with competitor pricing, supply and demand, or other external factors in the market depending on whether demand for a particular product is high or low. The concept of 'purchasing consortia' (Tella \& Virolainen, 2005), an arrangement between independent companies who join together to combine their individual requirements for goods to gain better prices, supply availability and assurance benefits than if each member purchased said goods alone is also illustrated.

To determine how services provided and consumed by agents both individually and collectively work in practice, we ran a MATLAB simulation for a period of seventy two hours to recreate real world demand fluctuations (where, as per our evaluation method, demand quantities were randomly generated; the following data were observed and 
stored for analysis: orders generated and served for each agent; joint orders generated and served; and price changes when demand goes high or low. At that point, we used the stored data to analyze how SCA autonomously discover services, manage relationships, and ensure trustworthiness in order to place their preferred orders.

\subsection{Orders Generated and Served}

When an existing supplier is unable to fulfill an order, SCN agents try to autonomously locate possible alternatives who can accommodate their preferences, ensure their trustworthiness, build relations and thereupon place orders ${ }^{3}$. A purchasing SCA will trade-off quantity against price of goods in reaching a decision, while the providing agent interacts with its own smart objects (e.g., shelves equipped with sensors) to locate ordered items and thereby service the purchaser.

In our simulation, a total of 33 orders containing all or some of the four featured products were generated. The customers and orders were generated randomly with different quantities for each product order according to on-hand stock insufficiencies. Exponential distribution was used to generate these because of its utility in modeling events that occur randomly over time (a process in which events occur continuously and independently at a constant average rate). In the case of other agents, for example the retailer who buys either directly from a manufacturer's distribution center or else from a wholesaler (who also buys their products from the manufacturer's distribution center) based on their stock insufficiencies from customer orders. Therefore, our demonstration reflects the number of possible agents in the $\mathrm{SCN}$ with the number of products identified as an example and applied the order fulfillment process as shown in Figure 4.

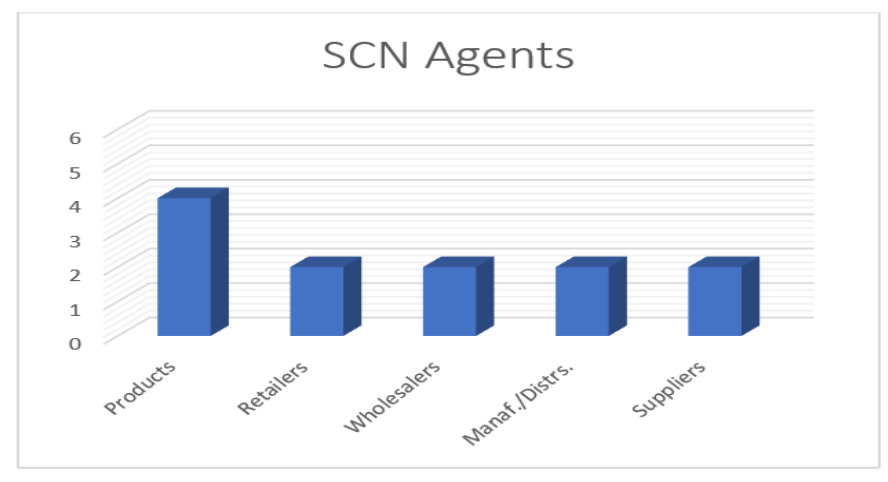

Figure 4. SCN Agents with Products

Specifically, fifteen orders from agent 'Retailer One' and 18 orders from agent 'Retailer Two' respectively were generated, as shown in Figures $5 \& 6$. These orders were then sent either to a manufacturer's distribution center or else to a wholesaler who buys their products from the manufacturer's distribution center. In the simulation agents (retailers) choose, based on their posed preferences (e.g., quantity and price) between agent distributor or wholesaler.

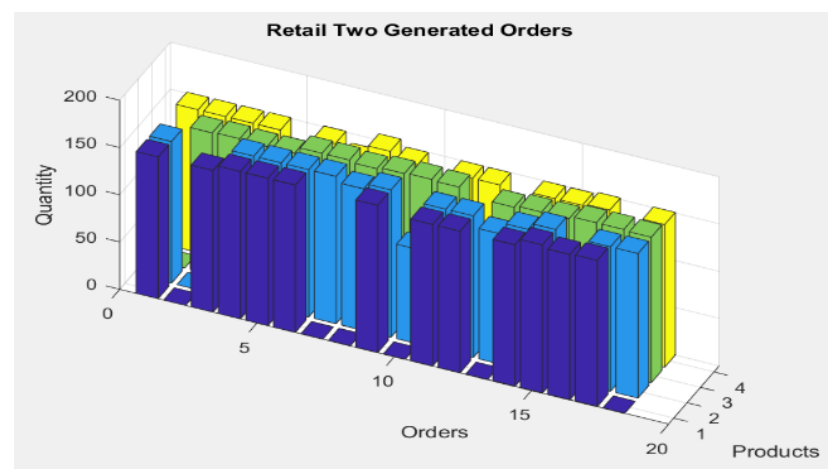

Figure 5. Generated Orders from Retailer One

\footnotetext{
${ }^{3}$ This encapsulates SIoT in practice; interactions between SCA and their 'own' smart objects represent the Internet of Things as we know it. In other words, the frontier between SIoT and IoT (in our present context) occurs when Supply Community Agents seek one another via a SCN, relaying instructions between their own smart objects.
} 


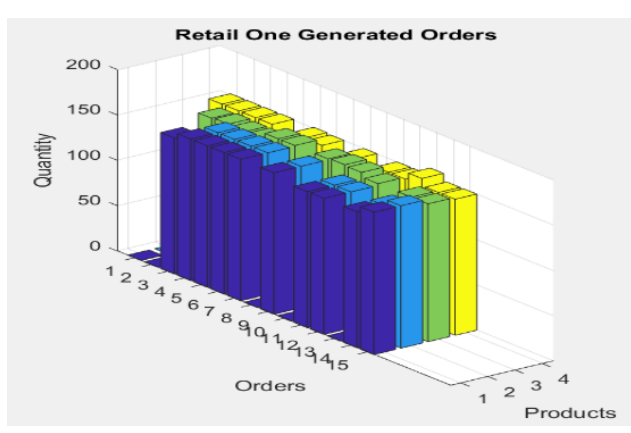

Figure 6. Generated Orders from Retailer Two

Each SCA (retailer) locates a potential agent (wholesaler and/or distributor) for their desired orders based on product quantity and price, as well as reliability; that is if an agent has previously sold the same goods and received a favourable rating from other agents, then a new relationship is built and the order placed. However, the 33 orders from agents (retailers) are each served by different wholesalers and distributors based on specific (retailer) requirements. Wholesalers and distributors then served these orders respectively using their SIoT functionalities to interact between objects. Agent (e.g., Retailer One) generated 15 orders but only 6 of them were sent to the Wholesaler, while the other 9 orders went to the Distributor as Figures 7 and 8 illustrate.

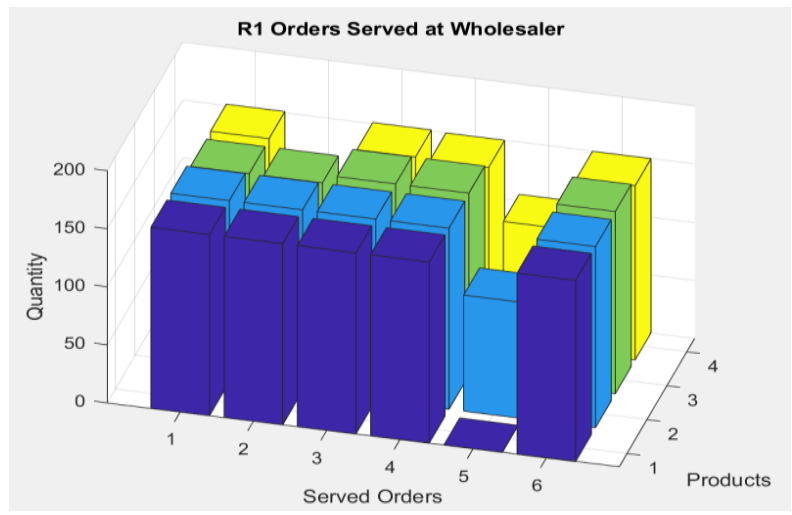

Figure 7. Retailer One Orders Served at Wholesaler

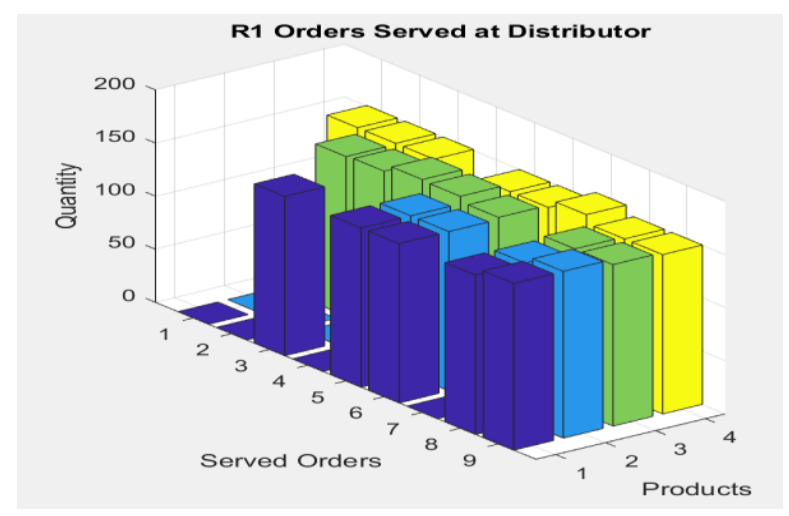

Figure 8. Retailer One Orders Served at Distributor

This is because each agent had access to the SCN and is able to locate possible agents who can accommodate their preferences. Thus, SIoT functionalities facilitated flexibility for SCA who can effectively work and interact with each other based on their respective needs. Therefore, the SCN enabled agents to find its desired needs from its friends or friend's friend in the network, but more significantly, delivered the possibility of discovering, selecting and using services an unknown agent within this SCN by using SIoT functionalities. 


\subsection{Joint Orders Generated and Served}

When agents are seeking to procure the same product(s) with the same or different quantities, etc. they may form a purchasing consortium (as described above) - i.e. place a joint order. In doing this agent consortium may try to locate possible agents who can accommodate their collective preferences. In that situation, we drew from the simulation 5 joint orders from agents (retailers) as Figure 9 illustrates.

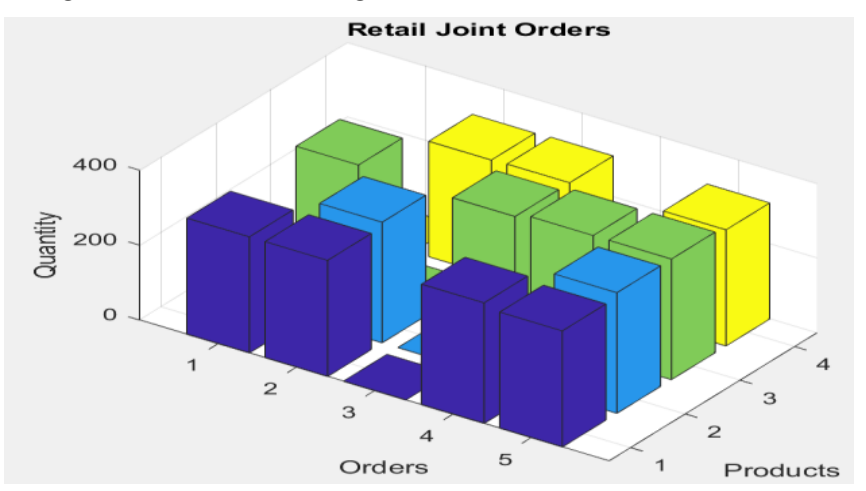

Figure 9. Generated Joint Orders

The same principles apply for joint orders as they do for single orders previously described. A consortium locates a potential agent (wholesaler and/or distributor) for their desired orders based on product quantity and price, as well as reliability; again, if an agent has previously sold the same goods and received a favourable rating from other agents, then a new relationship is built, trade off's made and the order placed. Thus, in our simulation the 5 joint orders are served by different agents (Wholesaler/Distributor) based on the preferences from agents (Retailers). Two orders out of five were sent to a Wholesaler agent able satisfy their preferences, while three were sent to the Distributor agent as Figures 10 and 11 illustrate.

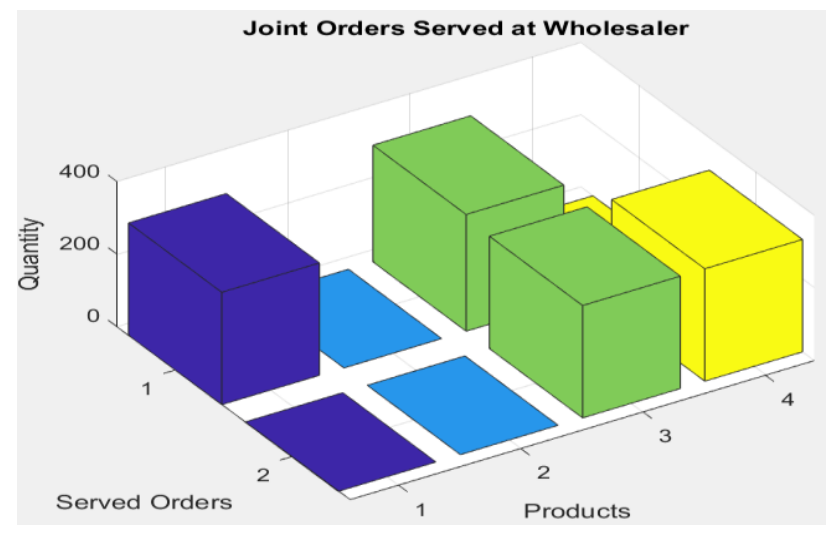

Figure 10. Joint Orders Served by Wholesaler

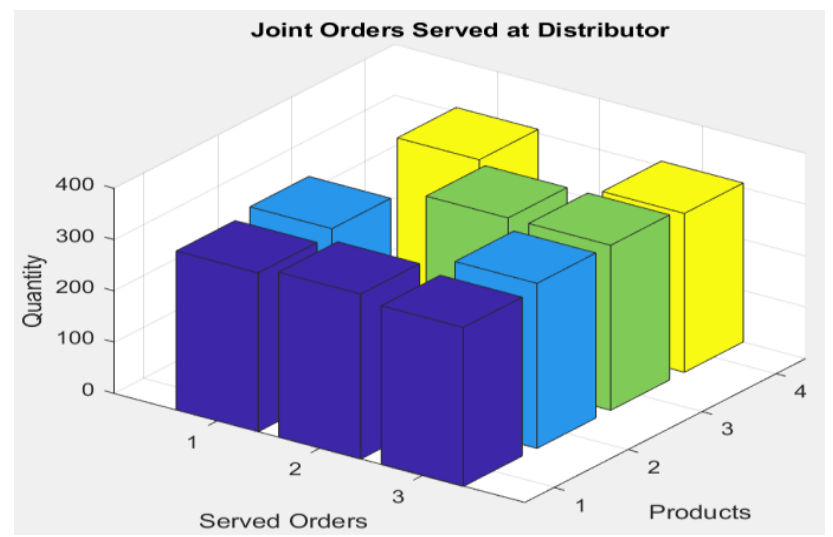

Figure 11. Joint Orders Served by Distributor 


\subsection{Product Demand and Price Changes}

Orders are sent to specified agents based on desired preferences. SIoT features allow SCA to identify other agents and select only those who could deliver on product preferences and trustworthiness. Consumers generate each product order, randomly oscillating from zero (0) to ten (10) at a time. In the simulation, the prices are determined based on utility and demand; if consumers demand more or less units of a product based on their needs then its price becomes 'elasticised'. Agents (e.g., retailers) evaluate offers for their products based on whether utility and demand is high or low; e.g., if the number of orders for one of the products on a single order is seven or more, then the price is discounted.

Consumers order different products at a time in quantities ranging from zero (0) to ten (10); as the orders arrive, an agent (e.g., Retailer One) checks which products are being ordered more or less so as to increase or decrease prices in line with demand. As shown in Figure 12 for instance, an agent (Retailer One) changed the prices of each of the products a number of times based on ordered quantities as it became clear that price was determined according to utility and demand.

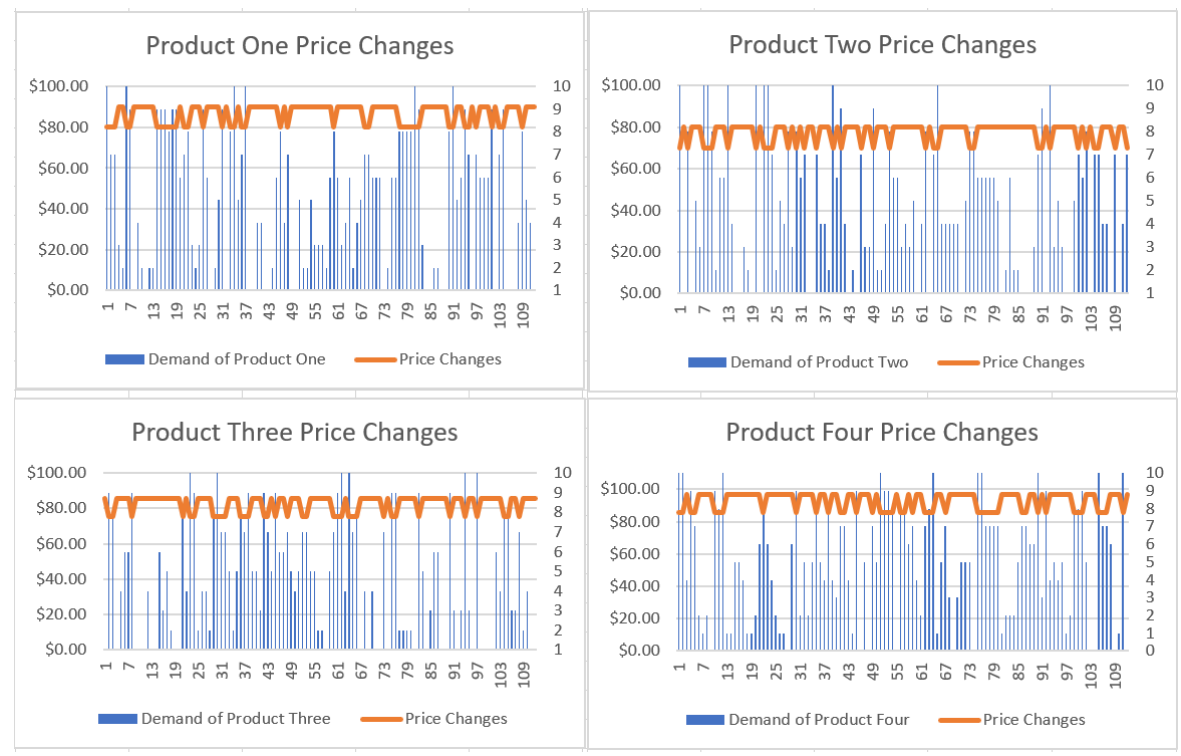

Figure 12. Retail One Product Demand and Price Changes

The agent (Retailer two) analyses the demand level based on orders received and then the price is increased or decreased. SIoT features and functionalities allowed sensing and detecting real-time information to make decisions based on demands and facilitate the smoothness of such operations. Also, evident is the agility that agents have to act on and respond quickly to fluctuations throughout the SCN.

On the other hand, if stock is insufficient, caused for example by a sudden unexpected order spike affecting the manufacturing agent, an internal scheduling agent updates the production agent which in turn may reprogram machinery (the reprogramming is again an example of IoT rather than SIoT). For instance, the agent (manufacturing) may have two production sites with different machines and a limit of 150 items for a product at a time. Consider a distributor agent who orders 16 times each with different quantities for all the products (se Figure 13). Once the (manufacturer) agent received the orders, a scheduler agent assigned to production site determines that the first order exceeds the production limit; thus the scheduler reprograms the production sites and balances the load to fulfil these orders as shown Figures 14 and 15. Also, the sensing layer and semantic engine sensed and detected real-time machinery conditions, for example, at production site one they reported that the machines cannot fulfil orders for product 4. In such case, production is rescheduled to site two. Likewise, it's been reported that product 1 also could not be fulfilled at site one and has to be rescheduled to site two for production. Whereas, production at site two on the other hand reported that it could not fulfil the production of product 3 and so the scheduler turned to site one to ensure smooth operation. Therefore, this allowed load balancing across sites as well as anticipating machine failures and other problems before they happened. 


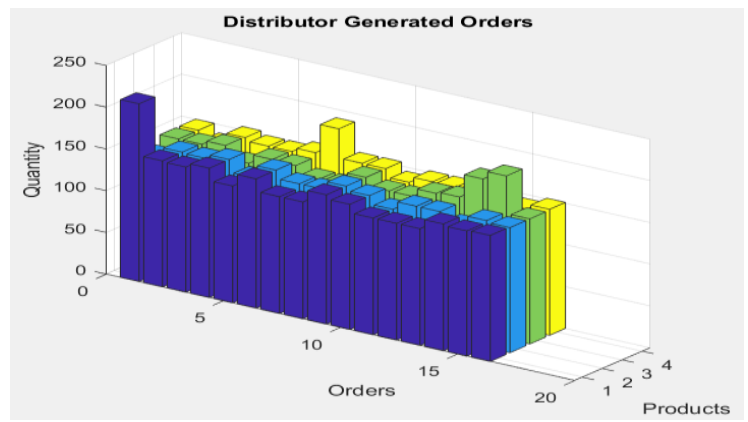

Figure 13. Generated Orders from Distributor

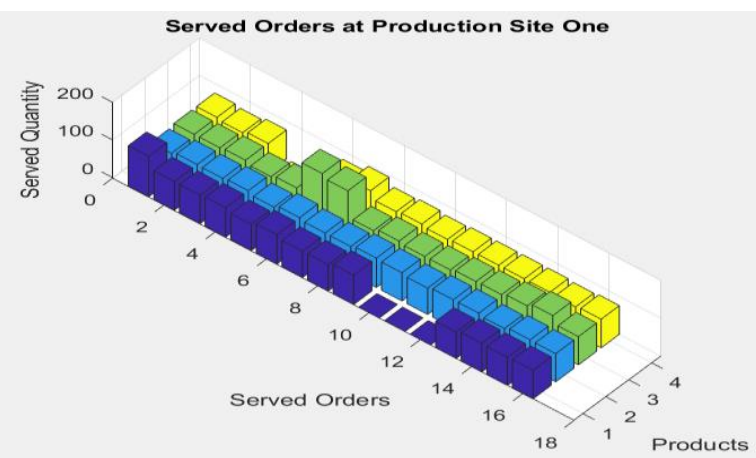

Figure 14. Orders Served Production Site One

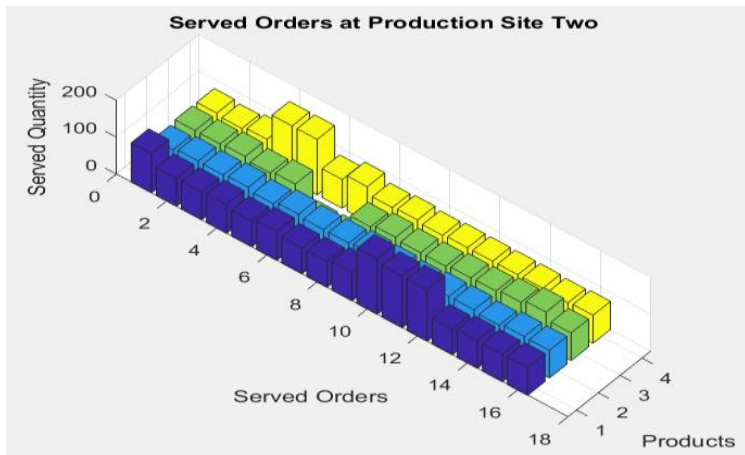

Figure 15. Orders Served production Site Two

SCA had access to the Supply Community Network to locate possible providers able to accommodate their preferences and to interact with them accordingly. This shows agents can find commodities/services based on their needs without human intervention. Such processes integrated the functional units across SCN to identify when and where. For instance, customers traded off store proximity against price, and quantity of goods in reaching a buy/not buy decision.

Using the service composition functionality, agents could then interact with other SCA to capture copious amounts of data, and more importantly to locate ordered items. By sending notifications about customer orders, after assembly they then prepare for shipment and communicate with a logistics agent to inform them of delivery requirements.

So, as the results show, SCA generated orders based on their requirements and preferences. Also, the result shows that agents can act on market dynamics so that e.g., if demand changes the price will change accordingly in quick response. Therefore, based on our simulation of SIoT capabilities we assert that the SCN framework is a practical way of meeting the challenges described in our introduction. 


\section{Conclusion}

A systematic literature review revealed existing work that provided a foundation for developing the notion of Supply Community Networks wherein agents (Supply Community Agents) assume humanoid social networking behavior. Concepts from the literature were identified as potential building blocks towards realizing SCN containing as they do, the basic SIoT components necessary to realize that aspiration together with a suitable system architecture.

The SCN framework includes smart objects with features and functionalities for the establishment and management of social relationships between SCA in such a way that they are navigable when integrated into a social network context. We also discussed an SIoT system architecture comprising a sensing layer, a network layer and an application layer; the architecture was described both from the server side and the object side in terms of its encompassing layers.

Supply Community Networks were purposely devised to address challenges posed by present day market dynamics. A feature of this is the way in which SCA will often look to service their own needs and to increase value and convenience for themselves using SIoT functionalities. The existing functions of smart objects were augmented by further methods specifically geared towards interaction within a Supply Community Network context.

To evaluate the proposed framework a scenario-based narrative was used in which SCN working practices were simulated to assess how SIoT orchestrated SCA work both individually and collectively. We substantiated our argument using MATLAB/Simulink to demonstrate how the services provided and consumed by agents both individually and collectively are enacted.

Our simulation has shown that the SIoT capabilities facilitate the flexibility of SCA allowing them to effectively work and deliver on SCN goals to discover, select and use appropriate network services. The simulation also yielded practical implications for the application of SCN agility in dealing with the afore-said market dynamics. SCA behavior was programmed to intentionally balance strong and weak ties and hence ensure reliability and flexibility within a SCN by virtue of loosely-coupled, flexible and interchangeable relationships among suppliers, customers and other agents.

The contribution of our work lies in demonstrating applicability of an existing Social IoT architecture to a new situation; we identified an appropriate technological basis for realizing the framework to test our theory of Supply Community Networks. Our work provides a novel solution to an emerging problem; that of managing and coordinating flows through a Supply Network with minimal human intervention and demonstrating the solution's efficacy in relation to that problem.

Some limitations can be identified; firstly, we only tested the order fulfilment process in terms of a single SCN due to the less common existence of integrated supply networks in the real world. Moreover, the issue of product returns was not considered (in part because of the baked goods case study in which returns would be unusual), although we recognize it is vital in creating a better customer experience. Furthermore, in the simulation, orders from SCA were randomly generated (not based on real-world data). The ultimate 'proof' of our approach defined would of course be substantiation that measurable improvements have resulted in the following areas: collection, analysis and proactive use of big data; inventory optimization; adaptability; rapid order fulfilment; customization \& scalability; sustainability; and regulatory compliance, visibility \& traceability (especially pertinent given that these are 'front burner' issues in food and beverage production). Whilst evaluation has already been extensive, there is no direct evidence of this as yet, simply because to collate and correlate such evidence requires prolonged industrial exposure, and in particular more elapsed time than is possible within the confines of a Doctoral programme. Nevertheless, conclusions from the research to date, and the level of interest shown by industry in Thailand suggest that it is not unrealistic to expect this level of contribution in the long run.

There are however additional matters outstanding. Our evaluation was restricted by the fact that we did not evaluate the entire conceptual framework using multiple SCNs. Instead we chose a simplistic example for baked goods employing a single Supply Community Network to provide proof of concept. In contrast, focal brands like Apple, Nike and Starbucks apportion success to having multiple supplier networks performing on a string. Their motivation is self-evident and can be summarized by five key drivers: profitability, cost management, customer satisfaction, flexibility and in particular globalization. Given the market dynamics set out in this paper, the primeval concept of 'supply' equating to delivery of goods on time has been steamrollered by a need to tailor and respond to divergent market expectations on pricing and packages. To do that, suppliers must embrace the capability and flexibility to personalize offerings based on multiple customer segmentation and richly diverse portfolios. 
A more detailed simulation involving multiple SCNs - one also addressing product returns, a vital component in overall customer satisfaction - therefore provides an opportunity to expand the literature base and build on findings of this paper. Given the mounting profile of SIoT among academics, and the gigantic leaps made by Internet of Things, it seems only a matter of time before industry latches on to the benefits of adopting this technology. One factor also to be considered however is that the framework presented here is by definition business critical inasmuch as any failure or disruption caused by said technology would cause turmoil for organizations and their operations. It is therefore recommended the literature be expanded to incorporate redundancy (fault tolerance) in the featured base architecture in pursuit of the 'holy grail' benchmark of five nines $(99.999 \%)$ availability. And yet notwithstanding future 'by-academics-for academics' investigation to swell the corpus on work further, academic collaboration with industrial partners remains key to achieve the ultimate measurable 'proof' discussed in our limitations above.

Finally, the implications for business practice are becoming clear; the paradigm shift that was IoT affected individuals, organizations, industries and society at large giving those leveraging its arsenal of capabilities competitive advantage through increased productivity, lowering of costs, timely new product/service offerings and the capability to re-imagine (in Tom Peters parlance) their business models. SIoT will take business to the next dimension; bolstering efficiency by driving down human intervention, whilst freeing organizations from the shackles of strong tie oriented rigid supply chains as we know them that both stifle innovation and foster continuity. Instead SIoT enabled weak tie SCNs will invert such outmoded concepts by stifling the missed opportunities of continuity, whilst simultaneously fostering innovation by albeit in reductionist terms transforming communication and the flow of information.

\section{References}

Atzori, L., Iera, A., \& Morabito, G. (2011). Siot: Giving a social structure to the internet of things. IEEE communications letters, 15(11), 1193-1195.

Atzori, L., Iera, A., Morabito, G., \& Nitti, M. (2012). The social internet of things (siot)-when social networks meet the internet of things: Concept, architecture and network characterization. Computer networks, 56(16), 3594-3608. https://doi.org/10.1016/j.comnet.2012.07.010

Blau, P. M. (1964 ). Exchange and power in social life. New York: Wiley.

Fiske, A. P. (1992). The four elementary forms of sociality: framework for a unified theory of social relations. Psychological review, 99(4), 689-723. https://doi.org/10.1037/0033-295X.99.4.689

Freeman, L. C., Roeder, D., \& Mulholland, R. R. (1979). Centrality in social networks: II. Experimental results. Social networks, 2(2), 119-141. https://doi.org/10.1016/0378-8733(79)90002-9

Ghobadi, S., \& D'Ambra, J. (2012). Knowledge sharing in cross-functional teams: a coopetitive model. Journal of Knowledge Management, 16(2), 285-301. https://doi.org/10.1108/13673271211218889

Girau, R., Nitti, M., \& Atzori, L. (2013). Implementation of an experimental platform for the social internet of things. Seventh International Conference on Innovative Mobile and Internet Services in Ubiquitous Computing (pp. 500-505). IEEE. https://doi.org/10.1109/IMIS.2013.90

Gordon, F. (2018, June 21). Iot Is Coming To The Supply Chain: What Happens Next? Retrieved October 8, 2018, from https://www.theinternetofthings.eu/gordon-feller-iot-coming-supply-chain-what-happens-next

Granovetter, M. S. (1973). The Strength of Weak Ties. American Journal of Sociology,(78): 1360-1380.. 1982. The Strength of Weak Ties: A Network Theory Revisited. In PV Marsden and N. Lin, (Eds.), Social Structure and Network Analysis (pp. 105-130). https://doi.org/10.1086/225469

Huynh, H. T., \& Soumare, I. (2011). Stochastic simulation and applications in finance with MATLAB programs. John Wiley \& Sons.

Kattan, P. I. (2008). Matlab For Beginners: A Gentle Approach. CreateSpace Publishing .

Kitchenham, B., Brereton, O. P., Budgen, D., Turner, M., Bailey, J., \& Linkman, S. (2009). Systematic literature reviews in software engineering-a systematic literature review. Information and software technology, 51(1), 7-15. https://doi.org/10.1016/j.infsof.2008.09.009

Li, Y., Zhang, S., \& Han, J. (2017). Dynamic pricing and periodic ordering for a stochastic inventory system with deteriorating items. Automatica , 76, 200-213. https://doi.org/10.1016/j.automatica.2016.11.003

Licklider, J. C. (2001, December 11). Topics for discussion at the forthcoming meeting, memorandum for: members and affiliates of the intergalactic computer network. Advanced Research Projects Agency. 
Retrieved October 16, 2018, from

http://www.kurzweilai.net/memorandum-for-members-and-affiliates-of-the-intergalactic-computer-network

Luo, Y. (2007). A coopetition perspective of global competition. Journal of world business, 42(2), 129-144. https://doi.org/10.1016/j.jwb.2006.08.007

Mack, S. (2018). The Basics of a Baked Goods Supply Chain. Retrieved August 20, 2019, from https://yourbusiness.azcentral.com/basics-baked-goods-supply-chain-25580.html

Moore, J. F. (1996). The death of competition: leadership and strategy in the age of business ecosystems. New York: HarperBusiness.

Nitti, M., Girau, R., \& Atzori, L. (2013). Trustworthiness management in the social internet of things. IEEE Transactions on knowledge and data engineering, 26(5), 1253-1266. https://doi.org/10.1109/TKDE.2013.105

Nitti, M., Roberto, G., Atzori, L., Iera, A., \& Morabito, G. (2012). A subjective model for trustworthiness evaluation in the social Internet of things. IEEE 23rd International Symposium on Personal Indoor and Mobile Radio Communications (PIMRC), (pp. 18-23). Sydney, NSW, Australia. https://doi.org/10.1109/PIMRC.2012.6362662

Omar, M., \& Mason, A. P. (2019). Supply Community Network: A Taxonomy of Flow Dimensions. Chinese Business Review, 18(7), 1-12.

Paulo, M. (2011). Social-driven Internet of connected objects. IAB workshop on Interconnecting Smart Objects with the Internet. Lisbon, Portugal.

Pieroni, M., Rizzello, L., Rosini, N., Fantoni, G., De Rossi, D., \& Mazzei, D. (2015). Affective Internet of Things: Mimicking human-like personality in designing smart-objects. IEEE 2nd World Forum on Internet of Things (WF-IoT) (pp. 400-405). IEEE. https://doi.org/10.1109/WF-IoT.2015.7389088

Porter, M. E. (1979). How competitive forces shape strategy. Harvard Business Review, 57(2), 137-145.

Rizk, N. (2002). Information technology and growth: prospects for Egypt's software industry. L'Égypte contemporaine, 93(465-466), 84-112.

Solis, B. (2015). Digital Darwinism: How Disruptive Technology Is Changing Business for Good. Wired. Retrieved 2, 2018.

Tella, E., \& Virolainen, V. M. (2005). Motives behind purchasing consortia. International Journal of Production Economics, 93 , 161-168. https://doi.org/10.1016/j.ijpe.2004.06.014

Turcu, C. E., \& Turcu, C. O. (2017). Social Internet of Things in Healthcare: From Things to Social Things in Internet of Things. In The Internet of Things: Breakthroughs in Research and Practice (pp. 88-111). IGI Global. https://doi.org/10.4018/978-1-5225-1832-7.ch005

\section{Copyrights}

Copyright for this article is retained by the author(s), with first publication rights granted to the journal.

This is an open-access article distributed under the terms and conditions of the Creative Commons Attribution license (http://creativecommons.org/licenses/by/4.0/). 